\title{
Arterial injuries after penetrating brain injury in civilians: risk factors on admission head computed tomography
}

\author{
Uttam K. Bodanapally, MBBS, ${ }^{1}$ Nitima Saksobhavivat, MD, ${ }^{2}$ \\ Kathirkamanathan Shanmuganathan, MD, ${ }^{1}$ Bizhan Aarabi, MD, ${ }^{3}$ and Ashis K. Roy, PhD ${ }^{1}$
}

Departments of ${ }^{1}$ Radiology and ${ }^{3}$ Neurosurgery, R Adams Cowley Shock Trauma Center, University of Maryland Medical Center, Baltimore, Maryland; and 2Department of Radiology, Faculty of Medicine Ramathibodi Hospital, Mahidol University, Bangkok, Thailand

OBJECT The object of this study was to determine the specific CT findings of the injury profile in penetrating brain injury $(\mathrm{PBI})$ that are risk factors related to intracranial arterial injuries.

METHODS The authors retrospectively evaluated admission head CTs and accompanying digital subtraction angiography (DSA) studies from patients with penetrating trauma to the head in the period between January 2005 and December 2012. Two authors reviewed the CT images to determine the presence or absence of 30 injury profile variables and quantified selected variables. The CT characteristics in patients with and without arterial injuries were compared using univariate analysis, multivariate analysis, and receiver operating characteristic (ROC) curve analysis to determine the respective risk factors, independent predictors, and optimal threshold values for the continuous variables.

RESULTS Fifty-five patients were eligible for study inclusion. The risk factors for an intracranial arterial injury on univariate analysis were an entry wound over the frontobasal-temporal regions, a bihemispheric wound trajectory, a wound trajectory in proximity to the circle of Willis (COW), a subarachnoid hemorrhage (SAH), a higher SAH score, an intraventricular hemorrhage (IVH), and a higher IVH score. A trajectory in proximity to the COW was the best predictor of injury (OR 6.8 and $p=0.005$ for all penetrating brain injuries [PBIs]; OR 13.3 and $p=0.001$ for gunshot wounds [GSWs]). Significant quantitative variables were higher SAH and IVH scores. An SAH score of 3 (area under the ROC curve [AUC] for all PBIs 0.72; AUC for GSWs 0.71) and an IVH score of 3 (AUC for all PBIs 0.65; AUC for GSWs 0.65) could be used as threshold values to suggest an arterial injury.

CONCLUSIONS The risk factors identified may help radiologists suggest the possibility of arterial injury and prioritize neurointerventional consultation and potential DSA studies.

http://thejns.org/doi/abs/10.3171/2014.9.JNS14679

KEY WORDS intracranial arterial injuries; penetrating traumatic brain injury; traumatic intracranial pseudoaneurysms; trauma

$\mathrm{A}$ RTERIAL injuries in penetrating brain injury (PBI) generally result from contact with a projectile or by transfer of kinetic energy from the projectile with resultant shock wave and cavitation or both. ${ }^{13}$ The reported incidence of traumatic intracranial arterial aneurysms (TICA) from surviving patients with PBI ranges from 3\%-42\%.1,2,4,6,8,10,11 The diagnosis of intracranial arterial injuries in penetrating trauma is important because such injuries have a propensity to lead to secondary brain injury, which worsens the primary injury. ${ }^{6}$ Secondary neurological injury from complications of arterial injuries usually results in hemorrhage and ischemia, which are major causes of clinical deterioration and death in patients who have experienced penetrating head injuries. ${ }^{6}$ Hemorrhagic complications include intraparenchymal, subarachnoid, or intraventricular hemorrhages, and infarctions can be either thrombotic or embolic in nature.

Literature consensus supports diagnostic angiography

ABBREVIATIONS AUC = area under the ROC curve; COW = circle of Willis; CTA = CT angiography; DSA = digital subtraction angiography; GSW = gunshot wound; $\mathrm{IVH}=$ intraventricular hemorrhage; $\mathrm{MCA}=$ middle cerebral artery; $\mathrm{PBI}=$ = penetrating brain injury; $\mathrm{ROC}=$ receiver operating characteristic; $\mathrm{SAH}=$ subarachnoid hemorrhage; TICA = traumatic intracranial arterial aneurysm.

SUBMITTED March 25, 2014. ACCEPTED September 29, 2014.

INCLUDE WHEN CITING Published online October 31, 2014; DOI: 10.3171/2014.9.JNS14679.

DISCLOSURE The authors report no conflict of interest concerning the materials or methods used in this study or the findings specified in this paper. 
in hemodynamically stable patients as soon as it is safe to perform. ${ }^{3}$ Digital subtraction angiography (DSA) is considered the standard of reference to detect and characterize intracranial arterial injuries after PBI. ${ }^{3}$ However, DSA is expensive, labor intensive, and potentially difficult to perform in a timely manner when equipment or qualified personnel are limited; hence, DSA is not the optimal diagnostic tool in every patient with PBI. ${ }^{12}$ The role of CT angiography (CTA) in the evaluation of patients with PBI has not been investigated. However, the use of CTA has been considered to be of limited diagnostic utility in the presence of metallic artifacts. ${ }^{6}$ Techniques to screen for arterial injuries and identify high-risk patients in order to prioritize neurointerventional radiology consultation would be helpful after PBI. The available literature mentions various risk factors for TICA based on the injury profile of the penetrating wound..$^{1,4,8}$ But these risk factors were derived from wartime TICAs more than a decade ago. The majority of the risk factors were determined via CT studies or plain radiographic assessment of entry wounds, exit wounds, and trajectories of the projectiles, and the vascular injuries were confirmed by conventional angiographies from subacute postinjury clinical phases. Identifying risk factors based on precise imaging characteristics on admission CT can validate the previous findings and potentially reveal new risk factors that can only be assessed by imaging in the acute phase of injury.

The objective of this study was to determine the specific CT findings of the injury profile in PBI that are risk factors related to intracranial arterial injuries.

\section{Methods}

The study complied with the Health Insurance Portability and Accountability Act, and we obtained permission from our institutional review board to perform the study at our Level I trauma center. We conducted a retrospective review of a trauma registry containing records for 6110 adults with penetrating trauma. Criteria for inclusion in this study were 1) penetrating trauma to the head, occurring between January 2005 and December 2012, with a total width calvarial fracture and projectile involvement of the brain parenchyma; 2) acquisition of brain CT images at the time of admission and cerebral four-vessel DSA studies during the course of hospitalization; and 3) an age $\geq 18$ years. Exclusion criteria were as follows: 1) intracranial injuries without penetration of the cranium, and 2) concomitant neck vessel injuries caused by either multiple entry wounds or the extracranial trajectory of a single entry wound.

\section{Variable Construction}

We analyzed the relationship of 30 study variables on admission head CT images (Table 1). All of these variables were $\mathrm{CT}$ findings in patients with PBI. Risk factors identified in the literature were also included: facio-orbitopterional entry sites (Figs. 1-4), intracerebral hematoma (Fig. 2), shell fragments crossing midline structures (Figs. 4 and 5), wound profile crossing dural compartments, retained fragments, absence of an exit wound (penetrating wound as opposed to perforating wound), missile passage through Reil's triangle, and multiple shells and bone fragments scattered in paths that branch in various directions (Fig. 6) $1,2,4,8$

\section{Image Interpretation and Definitions}

Two trauma radiologists (K.S. and N.S.) with 20 and 2 years of experience, respectively, reviewed the CT images and agreed on findings by consensus. The reviewers were blinded to the DSA findings. The CT images included 5-mm axial sections. All studies were performed using 16-, 40-, or 64-MDCT scanners (Brilliance, Phillips Healthcare). The reviewers assessed and recorded the presence of all the CT variables of craniocerebral trauma. All of the variables were given nominal scores based on the presence (score of 1) versus the absence (score of 0 ) of each of the $\mathrm{CT}$ variables. For subarachnoid hemorrhage (SAH; Figs. 2A and 7), intraventricular hemorrhage (IVH; Figs. 4 left and 5), and parenchymal hematoma, apart from the qualitative analysis, a quantitative analysis was performed. Once these CT findings were identified, the maximum diameter of the parenchymal hematoma was measured perpendicular to the trajectory of the wound (Fig. 2A). Subarachnoid hemorrhage was quantified using the Hijdra scale with modifications (Table 2). ${ }^{9}$ The modification accounts for the hemorrhage over the cerebral hemispheres (Fig. 2) and posterior cranial fossa in addition to the blood in the basal cisterns and sylvian fissures (Fig. 7), and the score ranges from 0 to 18. The extent of IVH was quantified using a modified Graeb score, whereby a score of 0 (no blood), 1 (sedimentation, < 25\% filled), 2 (moderately filled; Fig. 5), and 3 (completely filled; Fig. 4 left) was given to each ventricle for a maximum possible score of 12.,9

To describe the entry and exit wounds of the projectiles, the cranium was divided into three regions (Fig. 8): frontobasal, which also included orbitofacial injuries; transtemporal, which included injuries of the pterion, temporal bone, and greater wing of the sphenoid bones; and "other," which included occipital, suboccipital, high parietal, and high frontal injuries. The injuries were further divided into perforating injuries (Figs. 5 and 6) when the profile included entry and exit wounds with a tract through the brain parenchyma, whereas penetrating injuries (Figs. 1 and 4) were defined as a projectile penetrating the parenchyma without an exit wound. Bihemispheric (Figs. 4 and 5) and monohemispheric (Figs. 1 and 2) trajectories were defined as the presence and absence, respectively, of bullet shrapnel crossing the supratentorial midline structures. To describe the injury pattern and location (trajectory of the projectile), the brain was divided into the anterior, middle, and posterior cranial fossa. Proximity to the circle of Willis (COW) was defined as the wound trajectory within 2 $\mathrm{cm}$ of the suprasellar cistern (Figs. 1 and 3), which is the mean length of the $\mathrm{M}_{1}$ segment. Any of the $\mathrm{CT}$ variables that could not be assessed because of metal artifacts were considered to be missing data. The number of instances in which each CT variable could not be assessed is shown in Table 1.

The reference standard for arterial injury was the originally reported interpretation of the DSA study performed during the hospital course. The reports were further confirmed by direct review of the images. 
TABLE 1. Comparison of CT variables between arterial injury and nonarterial injury groups with PBIs*

\begin{tabular}{|c|c|c|c|c|c|c|c|c|}
\hline \multirow[b]{2}{*}{ CT Variable } & \multicolumn{4}{|c|}{ All PBIs (55 patients) } & \multicolumn{4}{|c|}{ GSWs (43 patients) } \\
\hline & MD & Non-Al & Al & p Value $†$ & MD & Non-Al & $\mathrm{Al}$ & p Value $†$ \\
\hline \multicolumn{9}{|l|}{ Entry } \\
\hline Frontobasal & 0 & $12(35.3 \%)$ & $9(42.9 \%)$ & 0.57 & 0 & $9(33.3 \%)$ & $9(56.2 \%)$ & 0.14 \\
\hline Temporal & 0 & $13(38.2 \%)$ & $11(52.4 \%)$ & 0.3 & 0 & $10(37 \%)$ & $7(43.7 \%)$ & 0.66 \\
\hline Frontobasal-temporal & 0 & $25(73.5 \%)$ & $20(95.2 \%)$ & 0.04 & 0 & $19(70.4 \%)$ & $16(100 \%)$ & 0.016 \\
\hline Other & 0 & $9(26.5 \%)$ & $1(4.8 \%)$ & 0.04 & 0 & $8(29.6 \%)$ & 0 & 0.01 \\
\hline \multicolumn{9}{|l|}{ Exit } \\
\hline Frontobasal & 1 & $2(22.2 \%)$ & $4(57 \%)$ & 0.15 & 1 & $2(22.2 \%)$ & $4(57 \%)$ & 0.15 \\
\hline Temporal & 1 & $5(55.6 \%)$ & $4(50 \%)$ & 0.8 & 1 & $5(55.6 \%)$ & $4(50 \%)$ & 0.8 \\
\hline Frontobasal-temporal & 2 & $7(77.8 \%)$ & $8(100 \%)$ & 0.16 & 2 & $7(77.8 \%)$ & $8(100 \%)$ & 0.16 \\
\hline Other & 1 & $2(22.2 \%)$ & 0 & 0.18 & 1 & $2(22.2 \%)$ & 0 & 0.18 \\
\hline Penetrating injury & 0 & $25(73.5 \%)$ & $13(61.9 \%)$ & 0.36 & 0 & $18(66.6 \%)$ & $8(50 \%)$ & 0.28 \\
\hline Perforating injury & 0 & $9(26.5 \%)$ & $8(38.1 \%)$ & 0.36 & 0 & $9(33.3 \%)$ & $8(50 \%)$ & 0.28 \\
\hline \multicolumn{9}{|l|}{ Wound trajectory } \\
\hline Monohemispheric & 2 & $25(75.8 \%)$ & $8(40 \%)$ & 0.009 & 2 & $19(73 \%)$ & $5(33.3 \%)$ & 0.01 \\
\hline Bihemispheric & 2 & $8(24.2 \%)$ & $12(60 \%)$ & 0.009 & 2 & $7(26.9 \%)$ & $10(66.6 \%)$ & 0.01 \\
\hline Transtentorial & 2 & $4(12 \%)$ & $1(5 \%)$ & 0.38 & 2 & $3(11.5 \%)$ & $1(6.7 \%)$ & 0.6 \\
\hline Unilat anterior cranial fossa & 1 & $13(38.2 \%)$ & $11(55 \%)$ & 0.23 & 1 & $11(40.7 \%)$ & $9(60 \%)$ & 0.23 \\
\hline Unilat middle cranial fossa & 1 & $12(35.3 \%)$ & $10(50 \%)$ & 0.29 & 1 & $10(37 \%)$ & $9(60 \%)$ & 0.15 \\
\hline Posterior fossa: unilat or bilat & 1 & $11(32.3 \%)$ & $1(5 \%)$ & 0.019 & 1 & $8(29.6 \%)$ & 0 & 0.019 \\
\hline Unilat anterior \& middle fossa & 2 & $1(3 \%)$ & $1(5 \%)$ & 0.7 & 2 & $1(3.7 \%)$ & $1(6.7 \%)$ & 0.66 \\
\hline In proximity to COW $(<2 \mathrm{~cm})$ & 0 & $4(11.7 \%)$ & $10(47.6 \%)$ & 0.003 & 0 & $3(11.1 \%)$ & $10(62.5 \%)$ & 0.0004 \\
\hline Transventricular trajectory & 0 & $1(2.9 \%)$ & $3(14.3 \%)$ & 0.11 & 0 & $1(3.7 \%)$ & $3(18.7 \%)$ & 0.1 \\
\hline Midline shift $\geq 5 \mathrm{~mm}$ & 3 & $10(31.2 \%)$ & $8(40 \%)$ & 0.51 & 0 & $10(37 \%)$ & $6(37.5 \%)$ & 0.97 \\
\hline Effacement of basal cisterns & 4 & $7(21.9 \%)$ & $8(42.1 \%)$ & 0.12 & 1 & $7(25.9 \%)$ & $7(46.6 \%)$ & 0.17 \\
\hline $\mathrm{SAH}$ & 6 & $24(82.7 \%)$ & $20(100 \%)$ & 0.05 & 1 & $23(88.4 \%)$ & $16(100 \%)$ & 0.15 \\
\hline SAH score & 9 & $2.88 \pm 2.42$ & $4.73 \pm 2.3$ & 0.01 & 3 & $3.1 \pm 2.36$ & $4.93 \pm 2.46$ & 0.026 \\
\hline IVH & 5 & $5(16.7 \%)$ & $9(45 \%)$ & 0.02 & 0 & $4(14.8 \%)$ & $7(43.7 \%)$ & 0.03 \\
\hline IVH score & 5 & $0.6 \pm 1.92$ & $2.4 \pm 3.6$ & 0.02 & 0 & $0.55 \pm 1.96$ & $2.25 \pm 3.4$ & 0.027 \\
\hline \multicolumn{9}{|l|}{ Bone or bullet fragments } \\
\hline Displaced into parenchyma & 0 & $28(82.3 \%)$ & $18(85.7 \%)$ & 0.74 & 0 & $22(81.5 \%)$ & $16(100 \%)$ & 0.06 \\
\hline $\begin{array}{l}\text { Crossing dural compartments } \\
\text { (transdural injury) }\end{array}$ & 2 & $11(33.3 \%)$ & $9(45 \%)$ & 0.39 & 2 & $9(34.6 \%)$ & $8(53.3 \%)$ & 0.24 \\
\hline Paths branching in various directions & 0 & $6(17.6 \%)$ & $7(33.3 \%)$ & 0.18 & 0 & $6(22.2 \%)$ & $7(43.7 \%)$ & 0.13 \\
\hline \multicolumn{9}{|l|}{ Hematoma } \\
\hline Subdural & 7 & $18(62.1 \%)$ & $14(73.7 \%)$ & 0.4 & 2 & $17(65.4 \%)$ & $13(86.7 \%)$ & 0.13 \\
\hline Epidural & 8 & $2(7.1 \%)$ & $3(15.8 \%)$ & 0.34 & 2 & $2(7.7 \%)$ & $2(13.3 \%)$ & 0.55 \\
\hline Intraparenchymal & 4 & $16(51.6 \%)$ & $15(75 \%)$ & 0.09 & 3 & $16(64 \%)$ & $11(73.3 \%)$ & 0.54 \\
\hline Diameter in cm & 4 & $3 \pm 1.3$ & $2.5 \pm 2.05$ & 0.27 & 3 & $3 \pm 1.3$ & $2.3 \pm 1.77$ & 0.85 \\
\hline
\end{tabular}

\section{Statistical Analysis}

Baseline CT characteristics of patients with and without arterial injuries were compared using the MannWhitney U-test for continuous variables and the Pearson chi-square test for categorical variables. Analysis was performed on a per patient basis; some of the patients had multiple sites of injury. Patients with a TICA and a concurrent non-TICA injury were considered to have a TICA injury alone. All candidate predictor variables associated with arterial injuries in the univariate analysis were incorporated into a multivariate logistic regression model. To identify the optimal threshold values for the continuous variables, we performed receiver operating characteristic (ROC) curve analysis. For all analyses, a 2 -tailed p value of less than 0.05 was considered to indicate statistical significance. Statistical analysis was performed using JMP 

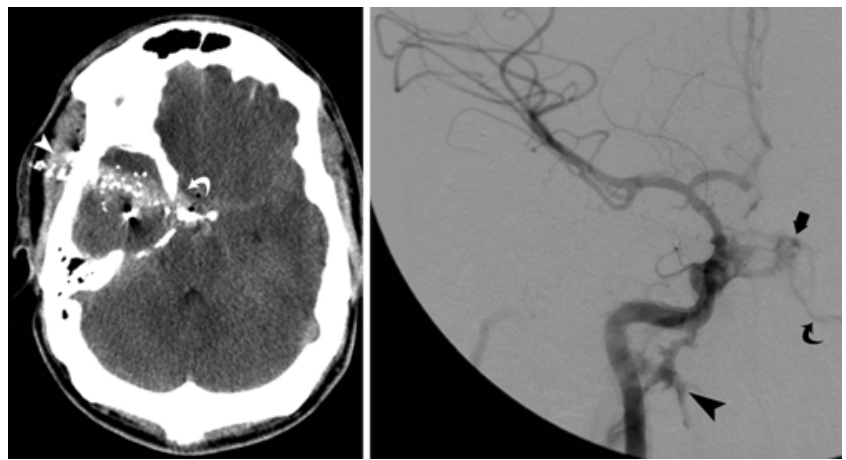

FIG. 1. Axial CT scan (left) obtained in an 18-year-old man with gunshot injury, demonstrating the variables of penetrating wound (no exit wound), entry wound over the pterional region (arrowhead), and trajectory of the wound close to COW (curved arrow). Coronal DSA image (right) demonstrating prompt opacification of the ipsilateral cavernous sinus and inferior petrosal sinus (arrowhead) after the infusion of contrast through the right internal carotid artery. Also visible is the opacification of the contralateral cavernous sinus (arrow) and superior petrosal sinus (curved arrow).

11 software (SAS Institute). Values are expressed as the means \pm standard deviation, unless indicated otherwise.

\section{Results}

Our retrospective review yielded 182 patients with cerebral or neck vessel angiograms obtained by the interventional neuroradiology section. Of these 182 patients, 55 had undergone DSA for suspected intracranial arterial injuries after having undergone admission head CT. These 55 consecutive patients formed the study group. Figure 9 shows the patient selection flowchart. There were 42 men and 13 women, with a mean age of 32 years (range 18-73 years). The mean period between the admission CT and the initial DSA study was 7.9 hours (range 90 minutes-18 hours). Twenty-five patients had follow-up angiograms, all obtained within 13 days of admission (mean 5 days, range 2-13 days). The mechanism of injury was gunshot wound (GSW) in 43 patients (shotgun blast in 3 of the 43), stabbing in 7 , and nail gun injury in 4 , and 1 patient was
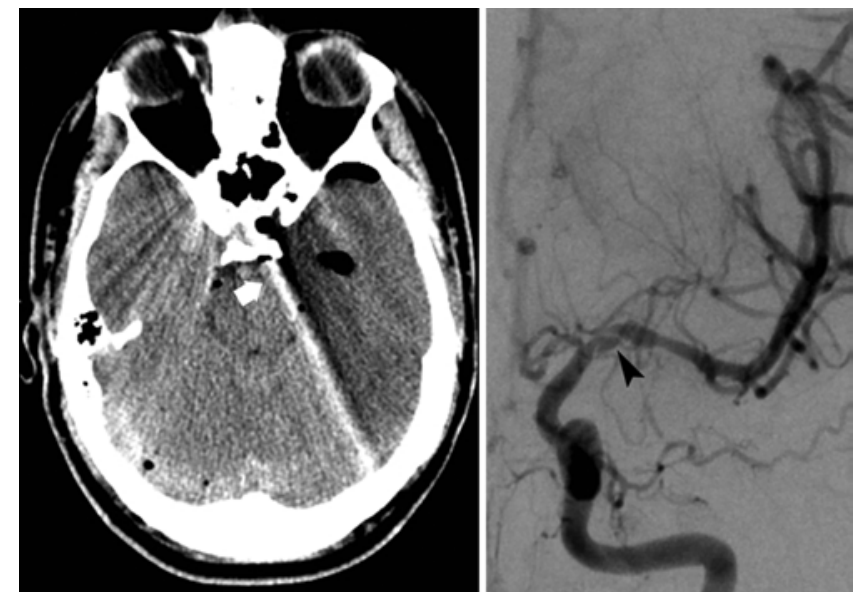

FIG. 3. Axial CT scan (left) obtained in a 29-year-old man with a gunshot injury, demonstrating the study variable of a trajectory close to the COW and a lodged bullet causing streak artifacts (arrow). A DSA image (right) demonstrates segmental irregularity and narrowing of the left middle cerebral artery (MCA) (proximal $\mathrm{M}_{1}$ segment) consistent with dissection (arrowhead).

impaled by a wooden piece. Twenty-one of the 55 patients with PBIs had arterial injuries. Five of these 21 patients had multiple injuries; 2 patients had 3 different sites of injury, and 3 patients had 2 sites of injury. Twenty-eight arterial injuries were identified: 11 TICAs (Fig. 2C), 8 dissections (Fig. 3 right), 6 occlusions (Fig. 4 right), and 3 carotid-cavernous fistulas (Fig. 1 right). Among the patients with GSWs (43), there were 16 with arterial injuries, and 7 of them had TICAs. The arterial injuries were located at the middle cerebral artery (MCA) (10), the internal carotid artery (8), the anterior cerebral artery (7), the ophthalmic artery (2), and the posterior inferior cerebellar artery (1).

After analyzing the missing data, we determined that the data were missing at random and that the missing variable was not dependent on the value of the variable. Missing data were dealt with by a pairwise deletion for univariate analysis and by a listwise deletion for multivariate analysis.
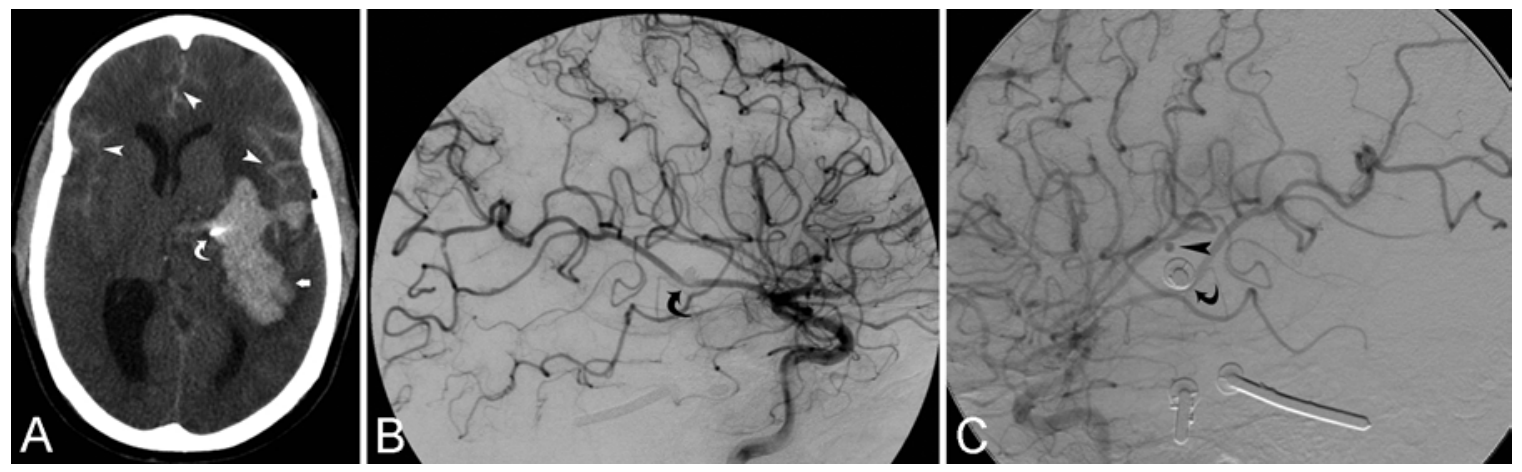

FIG. 2. Axial CT image (A) obtained in a 52-year-old man after nail gun injury, demonstrating parenchymal hematoma (arrow), $\mathrm{SAH}$ (arrowheads), and penetrating entry wound over the left temporal region. Also seen is the tip of projectile (curved arrow). Sagittal DSA image (B) obtained on the day of admission, showing a depressed angular branch of the left MCA due to the nail (curved arrow). Follow-up angiogram (C) obtained on Day 6, demonstrating the interval development of a pseudoaneurysm (arrowhead, traumatic intracranial arterial aneurysm) arising from the superior division of the left MCA and the depressed angular branch of the left MCA from the nail (curved arrow). Also visible in the image are two additional nails driven into the posterior fossa. 

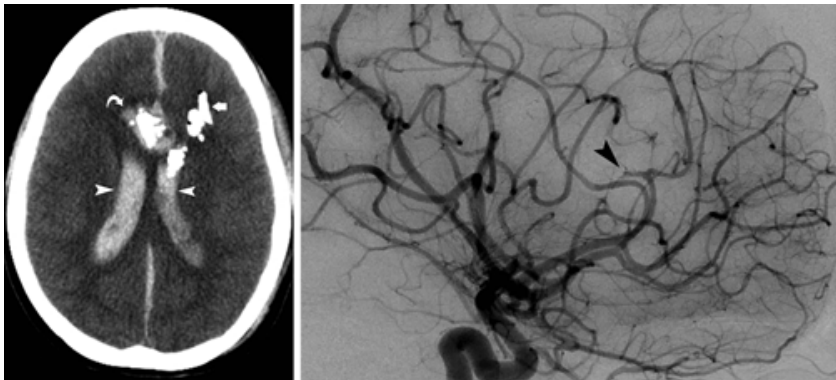

FIG. 4. Axial CT image (left) obtained in a 26 -year-old woman with a gunshot injury, demonstrating displaced bone fragments in the parenchyma (arrow), bihemispheric injury, and bone fragments crossing the dural compartments (curved arrow). Also visible is the study variable of intraventricular blood (arrowheads). A score of 3 was given to each of the lateral ventricles, as the blood completely filled the ventricles. A DSA image (right) demonstrates a truncated proximal pericallosal branch of the right anterior cerebral artery.

\section{Computed Tomography Variables in the Arterial Injury Cohort}

The results of each CT variable describing the wound profile are shown in Table 1. Patients were classified into an arterial injury group or a nonarterial injury group. The characteristics that differed between these two groups are shown in the table. The proportion of patients with an en-

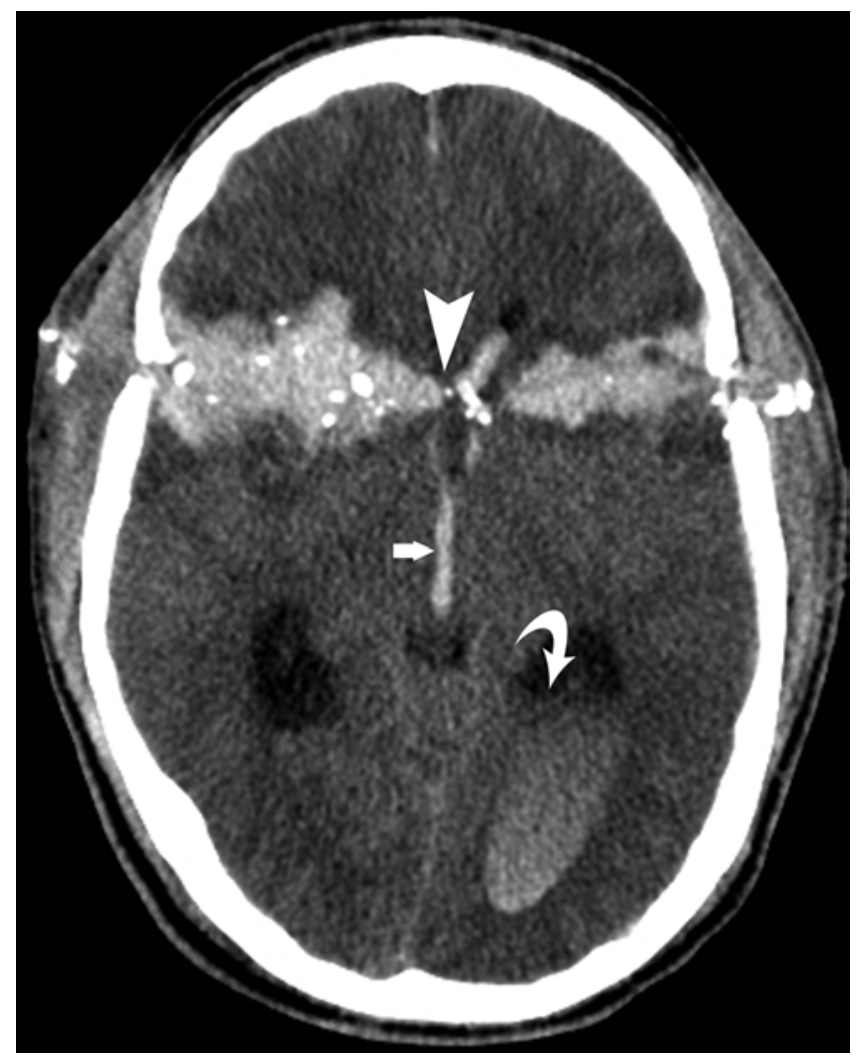

FIG. 5. Axial CT scan demonstrates perforating injury (presence of both entry and exit wounds), transventricular trajectory and transdural injury (arrowhead), bihemispheric trajectory, and intraventricular hemorrhage in a 19-year-old man with a gunshot injury. A score of 2 was given to each of the lateral ventricles, as they were moderately filled with blood (curved arrow), and a score of 3 was given to the third ventricle, as it was completely filled with blood (arrow).

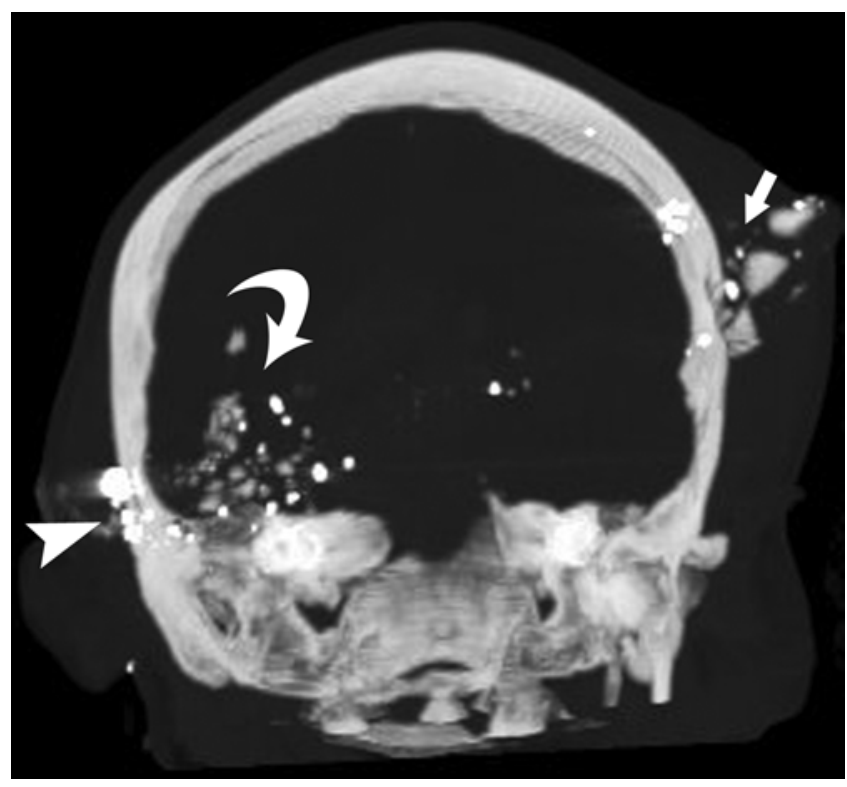

FIG. 6. Coronal maximum intensity projection CT scan demonstrates bone or bullet fragment paths branching in various directions (curved arrow), temporal entry site (arrowhead), and perforating injury with exit site over the parietal region (arrow) in a 29 -year-old man with a gunshot injury.

try wound over the frontobasal-temporal regions, a wound trajectory involving bilateral hemispheres, a wound trajectory in proximity to the $\mathrm{COW}$, an $\mathrm{SAH}$, a higher $\mathrm{SAH}$ score, an IVH, or a higher IVH score was significantly higher in the group with arterial injuries. The proportion of patients with entry wounds located over the occipital, high parietal, and frontal bones; a trajectory involving the posterior compartment; and a monohemispheric wound trajectory was significantly lower in the arterial injury group. Similar analysis exclusively in patients with GSWs showed identical results, except for the CT variable of SAH, which did not show a statistically significant difference between the arterial and nonarterial injury groups. The quantitative $\mathrm{CT}$ variables that had a significantly higher incidence in patients with an arterial injury were a higher SAH score $(4.73 \pm 2.3$ vs $2.88 \pm 2.42, \mathrm{p}=0.01$ for all PBIs and $4.93 \pm 2.46$ vs $3.1 \pm 2.36, p=0.03$ for GSWs) and a higher IVH score $(2.4 \pm 3.6$ vs $0.6 \pm 1.92, p=0.02$ for all PBIs and $2.25 \pm 3.4$ vs $0.55 \pm 1.96, p=0.027$ for GSWs). In the ROC curve analysis, an SAH score of 3 (area under the ROC curve [AUC] for all PBIs 0.72; AUC for GSWs 0.71) and an IVH score of 3 (AUC for all PBIs 0.65; AUC for GSWs 0.65) could be used as threshold values to suggest an arterial injury.

\section{Computed Tomography Variables in the TICA Cohort}

Analysis exclusively in patients with TICAs (11 patients) revealed a single CT variable (SAH score) that had a significantly higher incidence in patients with arterial injuries. Patients with a TICA had a higher SAH score $(5.2 \pm 2.5$ vs $3.2 \pm 2.3, p=0.03$ for all PBIs and $5.7 \pm 2.7$ vs $3.4 \pm 2.3$, $\mathrm{p}=0.03$ for GSWs). An SAH score of 6 (AUC 0.71) and 7 (AUC 0.73 ) could be used as threshold values to suggest a TICA in the PBI group and GSW group, respectively. 


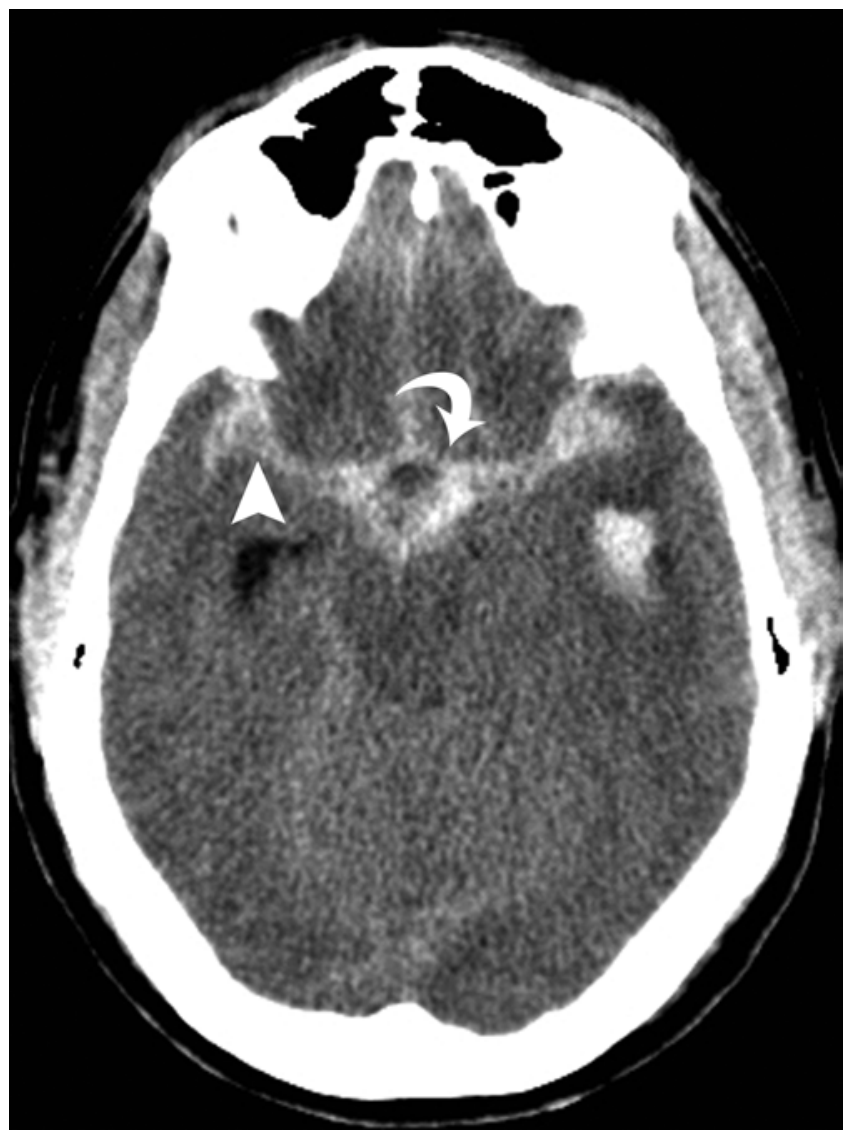

FIG. 7. Axial CT image demonstrates SAH in a 52-year-old man after a stab wound. A score of 3 was given to the basal cisterns, as they were completely filled with blood (curved arrow), and a score of 6 was given to the sylvian fissures, as they were completely filled with blood on both sides (arrowhead).

\section{Multivariate Logistic Regression}

There was a mild to moderate correlation between the variables included in the regression analysis (correlation coefficient range -0.51 to 0.45 ). According to a stepwise logistic regression analysis, a wound trajectory in proximity to the COW was the most reliable factor suggesting an arterial injury $(\beta=0.96,95 \%$ CI $0.31-1.68, \mathrm{SE} 0.34, \mathrm{OR}$ 6.8 , and $\mathrm{p}=0.005$ for all PBIs; $\beta=1.29,95 \%$ CI 0.55-2.16, SE 0.4, OR 13.3, and $\mathrm{p}=0.001$ for GSWs).

\section{Discussion}

In this study, we systematically analyzed the CT variables of craniocerebral injury patterns in patients with PBI and determined the risk factors related to the presence

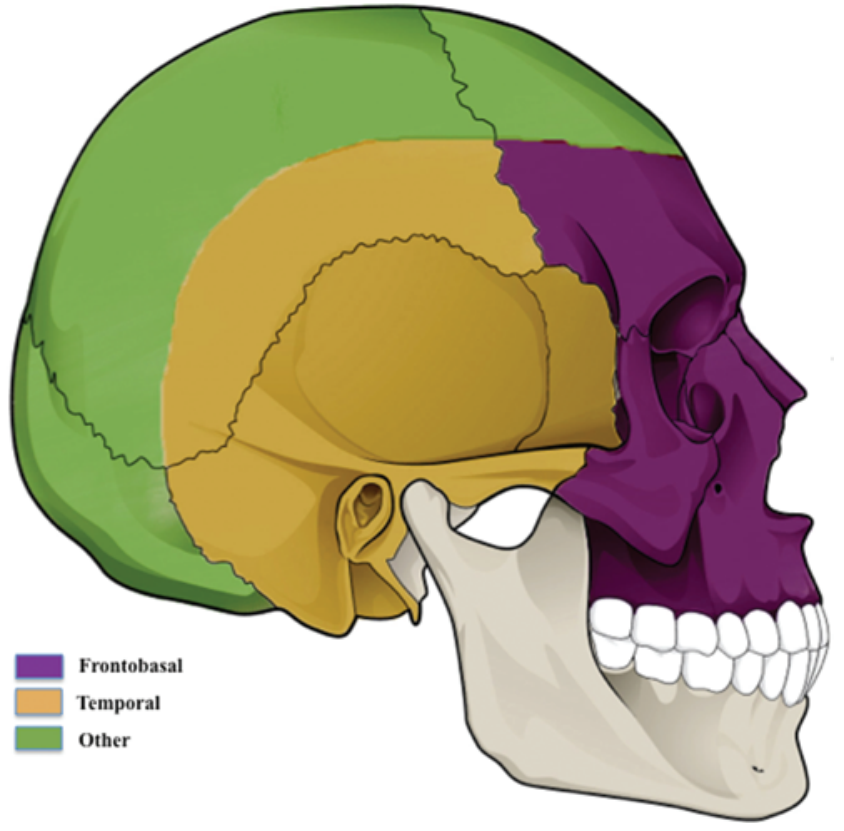

FIG. 8. Image showing the anatomical demarcation of the three entry and exit site zones used in the CT analysis. Figure is available in color online only.

of underlying arterial injuries. According to our results, an entry wound over the frontobasal-temporal regions, a wound trajectory involving bilateral hemispheres, a wound trajectory in proximity to the $\mathrm{COW}$, an $\mathrm{SAH}$, a higher SAH score, an IVH, and a higher IVH score were risk factors for arterial injuries. Patients with monohemispheric wound trajectory; entry wounds located over the occipital, high parietal, or high frontal bones; or a trajectory involving the posterior compartment were less likely to sustain an arterial injury. In the patient subgroup with TICAs, the only risk factor identified was a higher SAH score. The derived risk factors include well-recognized and simpleto-assess CT variables from admission head CTs following PBI. Patients considered to have a high risk of arterial injury should be evaluated with angiography. Computed tomography angiography, as a noninvasive option, has become a standard for PBI evaluation; however, the accuracy of this modality has not been established in the literature. We support the view that all patients with PBI should be screened with CTA. Moreover, those patients with established CT risk factors should be further evaluated with the gold standard DSA study to rule out arterial injuries, regardless of a negative CTA.

Similar to the studies by Aarabi $^{1}$ and Amirjamshidi

TABLE 2. Subarachnoid hemorrhage score, according to the modified Hijdra scale

\begin{tabular}{|c|c|c|}
\hline Anatomical Location & Score & Amount of Blood in Cisterns \& Fissures \\
\hline Basal cisterns & $0-3$ & \multirow{2}{*}{$\begin{array}{l}0, \text { none; } 1, \text { small amount; } 2, \text { moderately filled; } 3 \text {, } \\
\text { completely filled }\end{array}$} \\
\hline Sylvian fissures & 0-3 (on each side) & \\
\hline Hemispheres & 0-3 (on each side) & \multirow{2}{*}{$\begin{array}{l}0 \text {, none; } 1 \text {, seen in } 1-5 \text { CT slices; } 2 \text {, seen in 6-12 } \\
\text { CT slices; } 3 \text {, seen in }>12 \text { slices }^{*}\end{array}$} \\
\hline Posterior cranial fossa & 0-3 (uni- or bilateral) & \\
\hline Total score & $0-18$ & \\
\hline
\end{tabular}

\footnotetext{
* The CT slices were $5 \mathrm{~mm}$.
} 


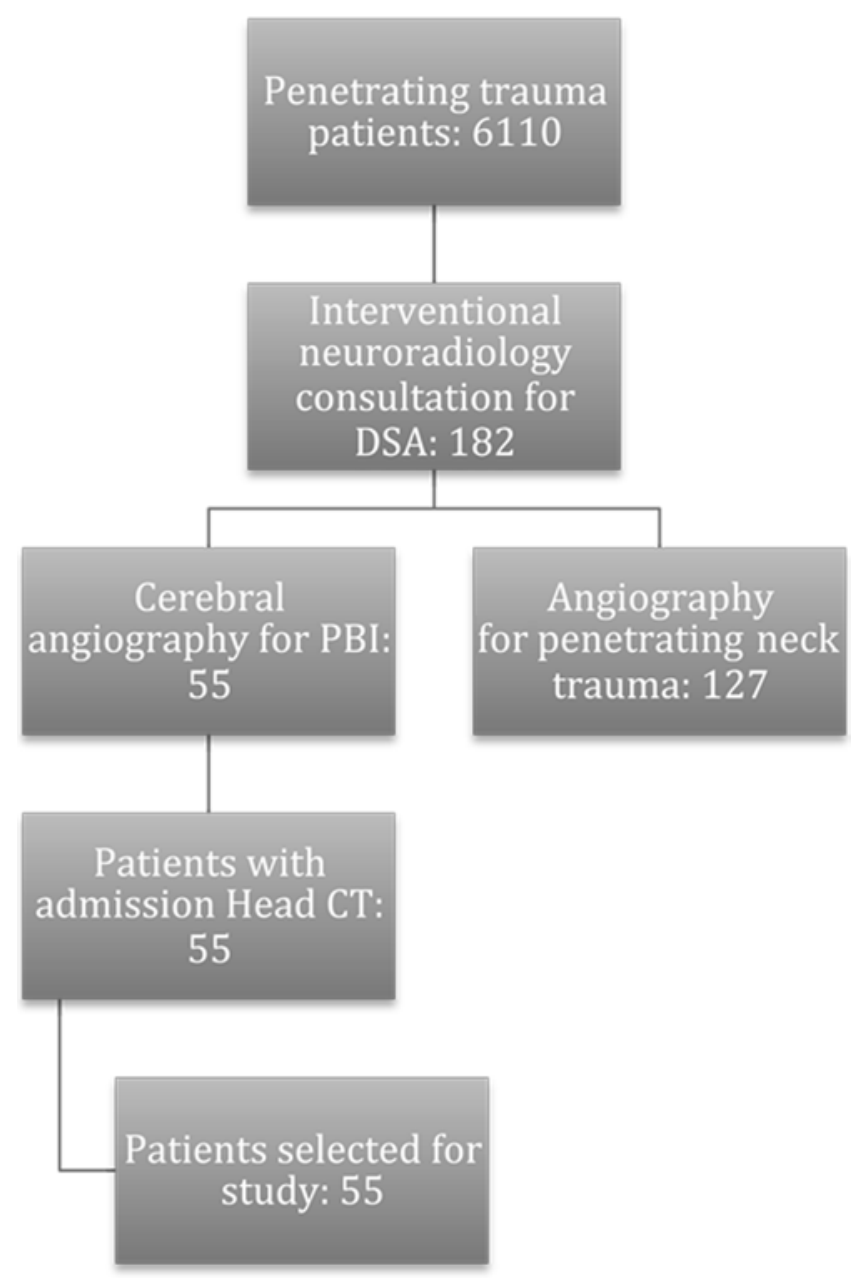

FIG. 9. Flowchart of patient selection.

et al., ${ }^{4}$ our analysis showed a statistically significant increased risk of arterial injuries in patients with frontobasal (frontal, facial, orbital) pterion (entire temporal and low parietal) entry wounds or a bihemispheric wound trajectory. However, in contrast to the authors cited above, we did not find a statistically significant difference between intracranial hematoma, projectiles crossing the dural compartments, mode of injury (penetrating vs perforation), multiple bullet or bone fragments scattered in paths that branch in various directions, and arterial injury or TICA. Bihemispheric injury (shrapnel crossing midline structures) is a form of transdural injury and was statistically significant. However, the CT variable transdural injury had no significant association with arterial injuries. This finding can be explained by the fact that transdural injuries also include the transtentorial wound trajectory, which is not associated with arterial injuries, making the whole group of transdural injuries a nonsignificant variable. Even the two CT variables that showed concordance with the established risk factors were not significant in the analysis performed exclusively in patients with TICAs.

We identified a number of new risk factors related to arterial injuries and TICA. A wound trajectory in proximity to the COW $(<2 \mathrm{~cm})$, an SAH, an IVH, a higher SAH score, and a higher IVH score are all newly identified risk factors shown to be associated with arterial injuries. The SAH score is a new, and the only, CT variable that had predictive significance with TICAs. A wound trajectory in proximity to the COW was the best predictor of arterial injury, as shown by logistic regression analysis. The odds ratios indicated that patients with such a trajectory are more than 7 times more likely to have an arterial injury among those with any PBIs and 13 times more likely to have an arterial injury among those with GSWs.

The reason for the discrepancy between the results from Aarabi and Amirjamshidi et al. and our results may be related to the difference in the number of patients included in our cohort, the difference in the mechanism of injury, the postinjury phase of evaluation, and perhaps from the precise localization of wounds given the availability of CT. All previous reports have involved wartime injuries from subacute postinjury clinical phases. Battlefield injuries differ from civilian injuries in that intracranial vascular injuries result from blast phenomenon and high-energy penetrating shrapnel, as opposed to low-energy bullet injuries in civilian cases. ${ }^{5}$ Moreover, the dynamic nature of traumatic arterial lesions may have resulted in the evolution of these injuries, and hence an accurate comparison of our results from acute-phase civilian injuries and the previously reported findings from delayed-phase wartime injuries may not be possible.

Our study has several limitations. First, its design is retrospective. Second, some significant CT variables may have been excluded from analysis, as CT variable selection was performed based on the previously identified risk factors, and some of the variables were selected by the study team members based on their clinical experience. The third limitation is the consensus interpretation of the CT findings. Consensus hardly reflects clinical practice, and mere consensus does not imply the correctness of a diagnostic decision in the absence of a reference standard for all the evaluated CT variables. Finally, the small study cohort of a civilian population may limit the generalizability of the conclusions.

\section{Conclusions}

Radiologists might suggest the possibility of intracranial arterial injuries based on admission head CT findings after PBI. Patients with such injuries should be directed toward an early neurointerventional consultation and potential DSA, despite a negative CTA study. However, future validation of these results from a large civilian PBI cohort is necessary.

\section{Acknowledgment}

We thank Brigitte Pocta for reviewing the manuscript.

\section{References}

1. Aarabi B: Management of traumatic aneurysms caused by high-velocity missile head wounds. Neurosurg Clin N Am 6:775-797, 1995

2. Aarabi B: Traumatic aneurysms of brain due to high velocity missile head wounds. Neurosurgery 22:1056-1063, 1988

3. Aarabi B, Alden T, Chestnut R: Vascular complications of penetrating brain injury. J Trauma 51 (2 Suppl):S26-S28, 2001 
4. Amirjamshidi A, Rahmat H, Abbassioun K: Traumatic aneurysms and arteriovenous fistulas of intracranial vessels associated with penetrating head injuries occurring during war: principles and pitfalls in diagnosis and management. A survey of 31 cases and review of the literature. J Neurosurg 84:769-780, 1996

5. Armonda RA, Bell RS, Vo AH, Ling G, DeGraba TJ, Crandall B, et al: Wartime traumatic cerebral vasospasm: recent review of combat casualties. Neurosurgery 59:1215-1225, 2006

6. Bell RS, Vo AH, Roberts R, Wanebo J, Armonda RA: Wartime traumatic aneurysms: acute presentation, diagnosis, and multimodal treatment of 64 craniocervical arterial injuries. Neurosurgery 66:66-79, 2010

7. Graeb DA, Robertson WD, Lapointe JS, Nugent RA, Harrison PB: Computed tomographic diagnosis of intraventricular hemorrhage. Etiology and prognosis. Radiology 143:91-96, 1982

8. Haddad FS, Haddad GF, Taha J: Traumatic intracranial aneurysms caused by missiles: their presentation and management. Neurosurgery 28:1-7, 1991

9. Hijdra A, Brouwers PJ, Vermeulen M, van Gijn J: Grading the amount of blood on computed tomograms after subarachnoid hemorrhage. Stroke 21:1156-1161, 1990

10. Jinkins JR, Dadsetan MR, Sener RN, Desai S, Williams RG: Value of acute-phase angiography in the detection of vascular injuries caused by gunshot wounds to the head: analysis of 12 cases. AJR Am J Roentgenol 159:365-368, 1992

11. Levy ML, Rezai A, Masri LS, Litofsky SN, Giannotta SL,
Apuzzo ML, et al: The significance of subarachnoid hemorrhage after penetrating craniocerebral injury: correlations with angiography and outcome in a civilian population. Neurosurgery 32:532-540, 1993

12. Sliker CW, Shanmuganathan K, Mirvis SE: Diagnosis of blunt cerebrovascular injuries with 16-MDCT: accuracy of whole-body MDCT compared with neck MDCT angiography. AJR Am J Roentgenol 190:790-799, 2008

13. Steenburg SD, Sliker CW, Shanmuganathan K, Siegel EL: Imaging evaluation of penetrating neck injuries. Radiographics 30:869-886, 2010

\section{Author Contributions}

Conception and design: Bodanapally, Shanmuganathan, Aarabi. Acquisition of data: Bodanapally, Saksobhavivat. Analysis and interpretation of data: Bodanapally, Saksobhavivat, Shanmuganathan, Roy. Drafting the article: Bodanapally, Saksobhavivat, Shanmuganathan. Critically revising the article: Shanmuganathan, Aarabi. Reviewed submitted version of manuscript: Bodanapally, Saksobhavivat, Shanmuganathan, Aarabi. Approved the final version of the manuscript on behalf of all authors: Bodanapally. Statistical analysis: Roy.

\section{Correspondence}

Uttam K. Bodanapally, Department of Radiology, University of Maryland Medical Center, 22S Greene St., Baltimore, MD 21201. email: ubodanapally@umm.edu. 